\title{
Effective analysis of Iris Images for Iris Recognition System
}

\author{
Anuradha Shrivas \\ Disha Institute of Management and Technology, \\ Raipur, India
}

\author{
Preeti Tuli \\ Department of Computer Science and Engineering, \\ DIMAT, \\ Raipur, India
}

\begin{abstract}
This paper proposes an iris recognition algorithm based on iris images. It consists of five major steps i.e., iris acquisition, localization, normalization, feature extraction and matching. The inner pupil boundary is localized using Circular Hough Transformation. The technique performs better in the case of occlusions and images muddled by artifacts such as shadows and noise. The outer iris boundary is detected by circular summation of intensity approach from the determined pupil center and radius. The localized iris image is transformed from Cartesian to polar co-ordinate system to handle different size, variation in illumination and pupil dilation. Corners in the transformed iris image are detected using covariance matrix of change in intensity along rows and columns. All detected corners are considered as features of the iris image. For recognition through iris, corners of both the iris images are detected and total number of codes that are matched between the two images are obtained. The two iris images belong to the same person if the number of matched corners is greater than some threshold value.
\end{abstract}

Keywords: Biometrics, Circular Hough transform, Hamming Distance.

\section{INTRODUCTION}

Iris is gaining lots of attention due to its accuracy, reliability and simplicity as compared to other biometric traits. The human iris is an annular region between the pupil (generally darkest portion of the eye) and sclera. It has many interlacing minute characteristics such as freckles, coronas, stripes, furrows, crypts and so on. These minute patterns in the iris are unique to each individual and are not invasive to their users. These properties make iris recognition particularly promising solution to society.

The concept of automated iris recognition has been initially proposed by Flom and Safir [1]. Daugman [2] has used multiscale quadrature wavelets to extract texture phase structure information of the iris to generate a 2048 bit iriscode and compared the difference between a pair of iris representations by computing their Hamming distance via the XOR operator. Boles and Boashash [3] have calculated zero-crossing representation of 1-D wavelet transform at various resolution levels of a virtual circle on an iris image to characterize the texture of the iris. Wildes et al. [4] have represented the iris texture with a Laplacian pyramid constructed with four different resolution levels and has used the normalized correlation to determine whether the input image and the model image are from the same class. This paper proposes an iris recognition algorithm which consists of five major steps. These steps are iris acquisition, localization, normalization, feature extraction and matching. The detailed image acquisition strategy is given in next section. Section 3 presents localization of inner pupil boundary using Circular Hough Transformation. The technique performs better in case of occlusions and images muddled by artifacts such as shadows and noise. Thus the inner pupil boundary can be detected without any sort of preprocessing required on the captured iris image. The outer iris boundary can be detected by circular summation of intensity approach from the known pupil center and radius. Section 4 deals with the normalization of the iris image. In this section iris image is transformed from Cartesian to polar co-ordinate system to handle different size, variation in illumination and other factors. Features are extracted from the normalized polar image in Section 5. The corner points are detected for the database and query images by using covariance matrix and detected points are matched by using the hamming distance approach.

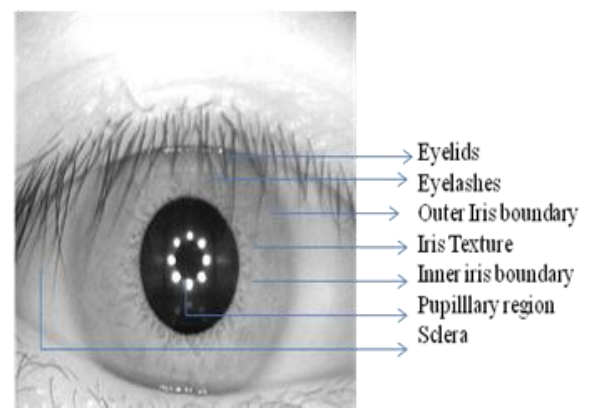

Figure1: Iris image

\section{IMAGE ACQUITION}

The iris image should be rich in iris texture as the feature extraction stage depends upon the image quality. Thus, the image is acquired by 3CCD camera placed at a distance of approximately $9 \mathrm{~cm}$ from the user eye. The approximate distance between the user and the source of light is about 12 $\mathrm{cm}$. Here the work is based on Iris images taken from standard CASIA data base.

\section{IRIS LOCALIZATION}

The acquired iris image has to be preprocessed to detect the iris, which is an annular portion between the pupil (inner boundary) and the sclera (outer boundary). The first step in iris localization is to detect pupil which is the black circular part surrounded by iris tissues. The center of pupil can be used to detect the outer radius of iris patterns. The important steps involved are:

1. Pupil detection

2. Outer iris localization 


\subsection{Pupil Detection}

The iris image is converted into grayscale to remove the effect of illumination. As pupil is the largest black area in the intensity image, its edges can be detected easily from the binarized image by using suitable threshold on the intensity image. But the problem of binarization arises in case of persons having dark iris. Thus the localization of pupil fails in such cases. In order to overcome these problems Circular Hough Transformation [5] for pupil detection can be used. The basic idea of this technique is to find curves that can be parameterized like straight lines, polynomials, circles, etc., in a suitable parameter space. The transformation is able to overcome artifacts such as shadows and noise. The approach is found to be good particularly dealing with all sorts of difficulties including severe occlusions [6].

The procedure first finds the intensity image gradient at all the locations in the given image by convolving with the sobel filters. The gradient images (Gvertical and GHorizontal) along $\mathrm{x}$ and $\mathrm{y}$ direction, is obtained by kernels that detect horizontal and vertical changes in the image. The sobel filter kernels are

$$
\begin{aligned}
& C_{\text {vertical }}=\left\{\begin{array}{llllllll}
-1 & -2 & -1 ; & 0 & 0 & 0 ; 1 & 2 & 1
\end{array}\right\} \\
& C_{\text {horizontal }}=\left\{\begin{array}{lllllll}
-1 & 0 & 1 ;-2 & 0 & 2 ;-1 & 0 & 1
\end{array}\right\}
\end{aligned}
$$

The absolute value of the gradient images along the vertical and horizontal direction is obtained to form an absolute gradient image using the equation

$$
G_{a b s}=G_{\text {Vertical }}+G_{\text {Horizontal }}
$$

where Gvertical is the convolution of image with Cvertical and Ghorizontal is the convolution of image with Chorizontal. The absolute gradient image is used to find edges using Canny [7]. The edge image is scanned for pixel $(\mathrm{P})$ having true value and the center is determined with the help of the following equations

$$
\begin{aligned}
& x c=x-r^{*} \cos (\theta) \\
& y c=y-r^{*} \sin (\theta)
\end{aligned}
$$

where $\mathrm{x}, \mathrm{y}$ are the coordinates at pixel $\mathrm{P}$ and $\mathrm{r}$ is the possible range of radius values, $\theta$ ranges from $[0: \pi]$.

For a particular value of $\mathrm{r}$, the values of $\mathrm{xc}$ and $\mathrm{yc}$ are obtained and stored in an accumlator and the accumulator counter is incremented every time the values of $\mathrm{xc}$ and $\mathrm{yc}$ satisfy image dimension criteria. The maximum value of accumulator acounter gives the centre of the pupil along with the radius as shown in Figure 2.
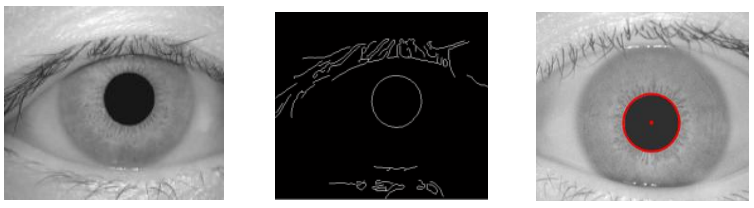

Figure 1 Steps involved in detection of inner pupil boundary

\subsection{Outer Iris Localization}

External noise is removed by blurring the intensity image. But too much blurring may dilate the boundaries of the edge or may make it difficult to detect the outer iris boundary, separating the eyeball and sclera. Thus a special smoothing filter such as the median filter [8] is used on the original intensity image. This type of filtering eliminates sparse noise while preserving image boundaries. After filtering, the contrast of image is enhanced to have sharp variation at image boundaries using histogram equalization as shown in Figure 3(a). This contrast enhanced image is used for finding the outer iris boundary by drawing concentric circles, as shown in Figure 3(b), of different radii from the pupil center and the intensities lying over the perimeter of the circle are summed up. Among the candidate iris circles, the circle having a maximum change in intensity with respect to the previous drawn circle is the iris outer boundary. Figure 3(c) shows an example of localized iris image.

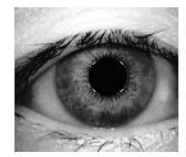

(a)

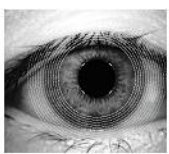

(b)

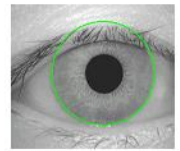

(c)
Figure 2 (a) Contrast enhanced image (b) Concentric circles of different radii (c) Localized Iris image

\section{IRIS NORMALIZATION}

Localizing iris from an image delineates the annular portion from the rest of the image. The concept of rubber sheet modal suggested by Daugman [2] takes into consideration the possibility of pupil dilation and appearing of different size in different images. For this purpose, the coordinate system is changed by unwrapping the iris and mapping all the points within the boundary of the iris into their polar equivalent as shown in Figure 4 . The mapped image has $80 \times 360$ pixels. It means that the step size is same at every angle. Therefore, if the pupil dilates the same points are picked up and mapped again which makes the mapping process stretch invariant [9]. Thus the following set of equations are used to transform the annular region of iris into polar equivalent

$$
\begin{aligned}
& I(x(\rho, \theta), y(\rho, \theta)) \rightarrow I(\rho, \theta) \\
& \text { with } \\
& x_{p}(\rho, \theta)=x_{\rho 0}(\theta)+r_{p} * \cos (\theta) \\
& y_{p}(\rho, \theta)=y_{\rho 0}(\theta)+r_{p} * \sin (\theta) \\
& x_{i}(\rho, \theta)=x_{i 0}(\theta)+r_{i} * \cos (\theta) \\
& y_{i}(\rho, \theta)=x_{i 0}(\theta)+r_{i} * \sin (\theta)
\end{aligned}
$$

where $\mathrm{rp}$ and ri are respectively the radius of pupil and the iris, while $(\operatorname{xp}(\theta), y p(\theta))$ and $(\operatorname{xi}(\theta), y i(\theta))$ are the coordinates of the pupillary and limbic boundaries in the direction $\theta$. The value of $\theta$ belongs to $[0 ; 2 \pi]$, $\rho$ belongs to $[0 ; 1]$. 

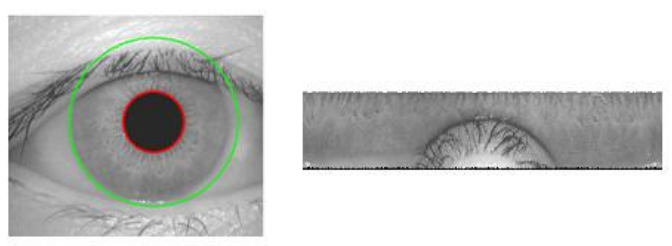

Figure 3 Iris normalization

\section{FEATURE EXTRACTION}

Corners in the normalized iris image can be used to extract features for distinguishing two iris images. The steps involved in corner detection algorithm are as follows

S1: The normalized iris image is used to detect corners using covariance matrix

S2: The detected corners between the database and query image are used to find cross correlation coefficient S3: If the number of correlation coefficients between the detected corners of the two images is greater than a threshold value then the candidate is accepted by the system

where $A_{\text {avg }}$ is the average of the area around point

Ii and $\sigma(\mathrm{A})$ is its standard deviation. Two corners are said to be co-related if cross correlation coefficient is greater than a given threshold value.

\subsection{Iris verification using corners}

Let $\mathrm{A}$ and $\mathrm{B}$ be the two iris images which are to be verified. Let $\mathrm{P}$ and $\mathrm{Q}$ be set of corners points detected in $\mathrm{A}$ and $\mathrm{B}$. For each point $\mathrm{p}$ in $\mathrm{P}$, let $\mathrm{m}$ points ( $\mathrm{q} 1 \ldots . . \mathrm{qm})$ in $\mathrm{Q}$ are less than $\mathrm{d}$ Euclidean distance from $\mathrm{p}$. Let $\mathrm{C} 1 \ldots \ldots . \mathrm{Cm}$ be cross correlation coefficient between $\mathrm{p}$ and ( $\mathrm{q} 1 \ldots \ldots \mathrm{qm})$. If maximum of $\mathrm{C} 1 \ldots . . . \mathrm{Cm}$ is greater than a threshold then $\mathrm{p}$ is said to be matched between A and B.

Let I1 and I 2 be the iris images of same person and I3 is another iris image to be verified. Let M1 be number of points matched between $\mathrm{I} 1$ and I 2 and M2 be number of corner points matched between I1 and I3.

The images I1 and I3 is said to belong to same person if the hamming distance between M1 and M2 is less than the threshold value.

$$
\frac{M_{1}-M_{2}}{M_{2}}<\psi
$$

where $\Psi$ is a threshold determined experimentally.

\section{EXPERIMENTAL RESULTS}

The work is carried on iris image CASIA database. Iris localization using Hough transform performs better as compared to other localization techniques in case of occlusion due to eyelids and eyelashes.

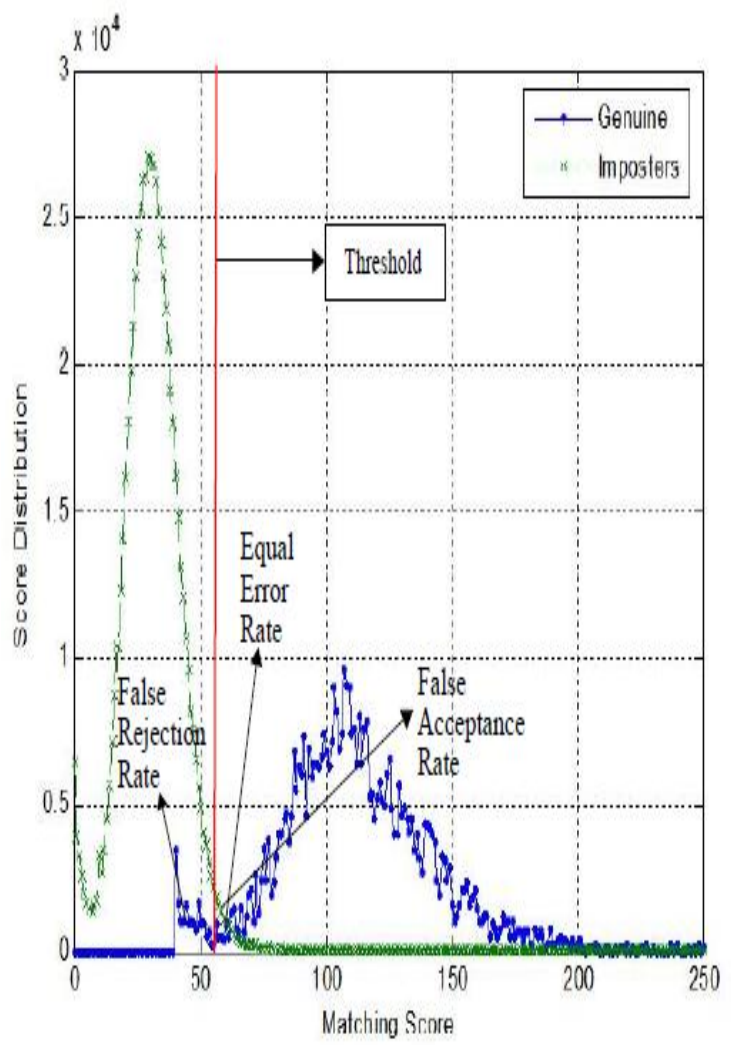

Figure5: Genuine matching score distribution

A public version of the CASIA Iris Database is available from sinobiometrics was of great help.

\section{REFERENCES}

[1]. L. Flom and A. Safir: Iris Recognition System. U.S. Patent No.4641394 (1987).

[2] J. G. Daugman: High confidence visual recognition of persons by a test of statistical independence. IEEE Transactions on Pattern Analysis and Machine Intelligence Vol. 15 (1993) 1148-1161.

[3] W.W. Boles, B. Boashah: A Human Identification Technique Using Images of the Iris and Wavelet Transform. IEEE Transaction on Signal Processing Vol. 46 (1998) 11851188 .

[4] R. Wildes, J. Asmuth, G. Green, S. Hsu, R. Kolczynski, J. Matey, S. McBride: A Machine-vision System for Iris Recognition. Machine Vision and Applications Vol. 9 (1996) $1-8$.

[5] T. Chuan Chen, K. Liang Chung: An Efficient Randomized Algorithm for Detecting Circles. Computer Vision \&Image Understanding Vol. 83 (2001) 172-191.

[6] E. R. Davies: Machine Vision. 3rd Edition: Elsevier (2005). 
International Journal of Science and Engineering Applications (IJSEA)

Volume 1 Issue 1, 2012

[7] J. Canny: A Computational Approach to Edge Detection. IEEE Transaction on Pattern Analysis and Machine Intelligence Vol. 8 (1986) 679-714.

[8] R. C. Gonzalez, R. E. Woods: Digital Image Processing. 2nd Edition, Pearson Education, India (2002).

[9]Y. Zhu, T. Tan, Y. Wang: Biometric Personal Identification Based on Iris Patterns. Proced dings of ICPR, International Conference on Pattern Recognition Vol. II (2000) 